\title{
A NOTE ON CONTINUOUS DEPENDENCE OF SOLUTIONS OF VOLTERRA INTEGRAL EQUATIONS
}

\author{
MATS GYLLENBERG
}

\begin{abstract}
The topologies induced by two families of seminorms on a vector space of functions $g: R^{+} \times R^{+} \times E^{n} \rightarrow E^{n}$ are compared. It is found that the continuous dependence of solutions of the Volterra equation $x(t)=f(t)+$ $\int_{0}^{t} g(t, s, x(s)) d s$ does not hold for the weaker topology. This result corrects an error in the book of Miller, Benjamin, Menlo Park, Calif., 1971.
\end{abstract}

The purpose of this paper is to give a counterexample to a proposition of $\mathbf{R}$. $\mathbf{K}$. Miller, concerning continuous dependence of solutions of Volterra integral equations. Consider the nonlinear Volterra equation

$$
x(t)=f(t)+\int_{0}^{t} g(t, s, x(s)) d s,
$$

where $f \in C\left(R^{+} ; E^{n}\right)$ (that is, $f$ is a continuous function from $R^{+}=[0, \infty)$ to the $n$-dimensional Euclidean space) and $g$ is a function defined on $R^{+} \times R^{+} \times E^{n}$ with values in $E^{n}$. In his book [2] Miller defines a vector space $\mathcal{G}$ of functions $g$ : $R^{+} \times R^{+} \times E^{n} \rightarrow E^{n}$ which satisfy certain hypotheses. Then he defines a family of seminorms on $\mathcal{G}$ (see (2) below) and proves, roughly speaking, that the solutions $x$ of (E) depend continuously on the functions $f$ and $g$, where the functions $f$ belong to $C\left(R^{+} ; E^{n}\right)$ topologized with the topology of uniform convergence on compact subsets and the functions $g$ belong to $\mathcal{G}$ topologized with the locally convex vector topology induced by the seminorms mentioned above (cf. Theorem II.4.2 and its corollaries [2, pp. 108-116]). Miller gives an alternative characterization of the seminorms (see (4) below). In this note I will prove that this characterization is erroneous and that the seminorms on the right side of (4) lead to a topology on $\mathcal{G}$ for which the continuity theorems in [2] do not hold.

The exact definition of $\mathcal{G}$ is given in [2, pp. 106-108]. For the purpose of this note it suffices to know that $\mathcal{G}$ contains all functions of the type

$$
g(t, s, x)=a(t-s) h(s, x),
$$

where $h$ is a continuous function $R^{+} \times E^{n} \rightarrow E^{n}$, and $a$ is a locally integrable real valued function which vanishes on $(-\infty, 0)$.

The seminorms on $\mathcal{G}$ are defined in the following way (cf. [2, p. 107]): For every $g \in \mathcal{G}$, every positive real number $K$ and every bounded set $B$ in $E^{n}, p(g, K, B)$ is defined by

$$
p(g, K, B)=\sup \left\{\int_{0}^{t}|g(t, s, \varphi(s))| d s \mid t \in[0, K], \varphi \in C([0, K] ; B)\right\},
$$

Received by the editors May 9, 1980.

AMS (MOS) subject classifications (1970). Primary 45D05.

Key words and phrases. Volterra integral equation, continuous dependence of solutions. 
where $C([0, K] ; B)$ is the set of all continuous functions on $[0, K]$ with values in $B$. The family of all these seminorms is denoted by $\mathscr{P}$, that is

$$
\mathscr{P}=\left\{p(\cdot, K, B) \mid K>0, B \text { bounded in } E^{n}\right\} .
$$

On pp. 107 and 145 (problem 16) of [2] Miller claims without proof that

$$
p(g, K, B)=\sup \left\{\int_{0}^{t}|g(t, s, x)| d s \mid t \in[0, K], x \in B\right\} .
$$

Denote the right side of (4) by $q(g, K, B)$ and let

$$
\mathcal{Q}=\left\{q(\cdot, K, B) \mid K>0, B \text { bounded in } E^{n}\right\} \text {. }
$$

It is clear that $\mathcal{Q}$ is a family of seminorms on $\mathcal{G}$.

I will now show that Miller's assertion (4) is false. I will show not only that in general $p(g, K, B) \neq q(g, K, B)$, but that the families $\mathcal{P}$ and $\mathcal{Q}$ in fact induce different topologies on $\mathcal{G}$.

It clearly suffices to restrict the proof to the one dimensional case $n=1$. For each positive integer $k$, let $h_{k}$ be a continuous function $R^{+} \times E^{1} \rightarrow E^{1}$ with the following properties:

$$
\begin{aligned}
0 \leqslant h_{k}(s, x) \leqslant 1 & \left(s \in R^{+}\right)\left(x \in E^{1}\right), \\
h_{k}(s, s)=1 & \left(s \in R^{+}\right), \\
h_{k}(s, x)=0 & \text { if }|s-x|>1 / 2 k .
\end{aligned}
$$

Define $g_{k}: R^{+} \times R^{+} \times E^{1} \rightarrow E^{1}$ by

$$
g_{k}(t, s, x)=a(t-s) h_{k}(s, x),
$$

where $a$ is the unit step function

$$
a(t)= \begin{cases}0 & \text { if } t<0 \\ 1 & \text { if } t>0 .\end{cases}
$$

Then $g_{k}$ belongs to $\mathcal{G}$ for each $k$. For every $K>0$ and every bounded set $B$ in $E^{1}$ one has

$$
\begin{aligned}
q\left(g_{k}, K, B\right) & \leqslant \sup \left\{\int_{0}^{K} h_{k}(s, x) d s \mid x \in B\right\} \\
& \leqslant \sup \left\{\int_{x-1 / 2 k}^{x+1 / 2 k} 1 d s \mid x \in B\right\}<\frac{1}{k} .
\end{aligned}
$$

This shows that $q\left(g_{k}, K, B\right) \rightarrow 0$ as $k \rightarrow \infty$ for every $K>0$ and every bounded $B \subset E^{1}$, which means precisely that $\left\{g_{k}\right\} \rightarrow 0$ in the topology $\tau_{2}$ induced by 2 .

On the other hand, let $K=1$ and $B=[0,1]$. Define $\varphi \in C([0,1] ;[0,1])$ by $\varphi(s)=s(s \in[0,1])$. Then

$$
\int_{0}^{1}\left|g_{k}(1, s, \varphi(s))\right| d s=\int_{0}^{1} h_{k}(s, s) d s=1
$$

for each $k \in N$. In particular, $p\left(g_{k}, 1,[0,1]\right)>1$ for each $k$ and thus $\left\{g_{k}\right\}$ does not converge to 0 in the topology $\tau_{\mathscr{\Phi}}$ induced by the family $\mathcal{P}$ of seminorms on $\mathcal{G}$.

Since $\left\{g_{k}\right\} \rightarrow 0$ in $\tau_{\mathscr{2}}$ but $\left\{g_{k}\right\} \nrightarrow 0$ in $\tau_{\odot}$, it is clear that $\tau_{\mathscr{\Phi}} \neq \tau_{\mathscr{2}}$. Since obviously

$$
q(g, K, B)<p(g, K, B)
$$


for every $g \in \mathcal{Q}, K>0$ and bounded $B$, the inclusion

$$
\tau_{\mathscr{Q}} \subset \tau_{\mathscr{P}}
$$

holds. I have now proved that $\tau_{\mathscr{Q}}$ is strictly weaker than $\tau_{\Phi \text {. }}$

Artstein has proved (cf. [1]) that $\tau_{\mathscr{\rho}}$ is not the weakest topology for which the continuous dependence holds and that, in fact, there is no weakest topology with this property. Thus the possibility that the continuous dependence holds for $\tau_{2}$ still exists. However, the following example shows that $\tau_{2}$ is too weak.

Let $\left\{g_{k}\right\}$ be the previously defined sequence in $\mathcal{G}$. As was shown above, $\left\{g_{k}\right\} \rightarrow 0$ in $\tau_{2}$. For each positive integer $k$, define $x_{k}$ by $x_{k}(t)=t\left(t \in R^{+}\right)$. Then

$$
\int_{0}^{t} g_{k}\left(t, s, x_{k}(s)\right) d s=\int_{0}^{t} h_{k}(s, s) d s=t=x_{k}(t)
$$

for each $t \in R^{+}$and each $k \in N$. Thus $x_{k}$ satisfies the equation

$$
x_{k}(t)=\int_{0}^{t} g_{k}\left(t, s, x_{k}(s)\right) d s
$$

on $R^{+}$for each $k \in N$. Trivially, $\left\{x_{k}\right\} \rightarrow x, x(t)=t$, in any topology, but $x$ is not a solution of the limit equation

$$
x(t)=\int_{0}^{t} 0 d s=0
$$

on any interval $[0, b]$. Thus Miller's theorem [2, II.4.2] is false if $\tau_{\mathscr{P}}$ is replaced by $\tau_{2}$

It should be stressed that Miller does not use his assertion $\tau_{\mathscr{P}}=\tau_{2}$ (which here has been proved incorrect) in the proof of theorem [2, II.4.2] and thus the counterexamples given in this note in no way affect the correctness of the theorem or its corollaries.

\section{REFERENCES}

1. Z. Artstein, Continuous dependence of solutions of Volterra integral equations, SIAM J. Math. Anal. 6 (1975), 446-456.

2. R. K. Miller, Nonlinear Volterra integral equations, Benjamin, Menlo Park, California, 1971.

Institute of Mathematics, Helsinki University of Technology, SF-02150 Espoo 15, FinLand 